\title{
Self-Organized GaN Quantum Wire UV Lasers
}

\author{
Heon-Jin Choi ${ }^{\dagger, \dagger}$ Justin C. Johnson, ${ }^{\dagger}$ Rongrui He, ${ }^{\dagger}$ Sang-Kwon Lee, ${ }^{\dagger, *}$ Franklin Kim, ${ }^{\dagger}$ \\ Peter Pauzauskie, ${ }^{\dagger}$ Joshua Goldberger ${ }^{\dagger}$ Richard J. Saykally, ${ }^{\dagger}$ and Peidong Yang ${ }^{*}, \dagger$, \\ Department of Chemistry, University of California, and Materials Science Division, \\ Lawrence Berkeley National Laboratory, Berkeley, California 94720
}

Received: March 21, 2003; In Final Form: June 1, 2003

\begin{abstract}
Quantum wire lasers are generally fabricated through complex overgrowth processes with molecular beam epitaxy. The material systems of such overgrown quantum wires have been limited to $\mathrm{Al}-\mathrm{Ga}-\mathrm{As}-\mathrm{P}$, which leads to emission largely in the visible region. We describe a simple, one-step chemical vapor deposition process for making quantum wire lasers based on the $\mathrm{Al}-\mathrm{Ga}-\mathrm{N}$ system. A novel quantum-wire-in-opticalfiber (Qwof) nanostructure was obtained as a result of spontaneous $\mathrm{Al}-\mathrm{Ga}-\mathrm{N}$ phase separation at the nanometer scale in one dimension. The simultaneous excitonic and photonic confinement within these coaxial Qwof nanostructures leads to the first GaN-based quantum wire UV lasers with a relatively low threshold.
\end{abstract}

Quantum confinement of charge carriers in more than one dimension in quantum wires and quantum dots has been predicted to yield improved performance of semiconductor lasers, relative to conventional quantum well devices. ${ }^{1,2}$ The carrier confinement is expected to lead to reduced threshold currents and narrower spectral line widths. The potential advantages of the quantum wire lasers could make them ideal for a variety of applications that require coherent light sources with low power consumption and high-speed digital modulation capability. A major challenge, however, has been the development of the fabrication technology for preparing quantum wire heterostructures that are compatible with laser applications. Almost all quantum wire lasers reported thus far have been made through molecular beam epitaxy, microfabrication, and lithographical techniques on the GaAs/InP system for visible light emission. ${ }^{1,3,4}$ Significant technical hurdles exist for the direct fabrication of GaN-based quantum wire lasers, despite their obvious potential in short-wavelength photonic devices and hightemperature/high-power optoelectronics. ${ }^{5-8}$ Herein, we report the first realization of self-organized, monolithically singlecrystalline $\mathrm{GaN} / \mathrm{Al}_{x} \mathrm{Ga}_{1-x} \mathrm{~N}(x=0.75)$ core-sheath onedimensional (1D) nanostructures. Quantum wires of $\mathrm{GaN}$ (refractive index of 2.54) with diameters as small as $5 \mathrm{~nm}$ are cladded by $\mathrm{Al}_{0.75} \mathrm{Ga}_{0.25} \mathrm{~N}$ (refractive index of 2.25) sheaths with uniform thicknesses in the range of 50-100 nm, forming a novel quantum-wire-in-optical-fiber (Qwof) structure. As a manifestation of the quantum confinement, a blue shift of the photoluminescence (PL) has been observed. Moreover, the simultaneous excitonic and photonic confinement within these coaxial Qwof nanostructures leads to the first GaN-based quantum wire UV lasers with relatively low thresholds.

Recently, UV lasing has been demonstrated by our groups for the $\mathrm{ZnO}$ and $\mathrm{GaN}$ nanowire systems. ${ }^{9-11}$ In these earlier studies, the entire faceted nanowire (generally with a diameter of $>100 \mathrm{~nm}$ ) serves both as a gain medium and as a FabryPerot optical cavity. This nanowire nanolaser configuration

\footnotetext{
* Author to whom correspondence should be addressed. E-mail: p_yang@uclink.berkeley.edu.

$\dagger$ University of California.

$\doteqdot$ Lawrence Berkeley National Laboratory.
}

places a lower limit on the lasing nanowire diameter, approximately $\lambda / 2 n$ (where $n$ is the refractive index), below which the nanowire is no longer capable of sustaining even a leaky transverse mode; consequently, no lasing has been observed from free-standing $\mathrm{ZnO}$ and $\mathrm{GaN}$ nanowires with diameters of $<100 \mathrm{~nm}$. This limitation is obviously undesirable if one wants to explore beyond photonic confinement (waveguiding), e.g., strong excitonic confinement within such nanostructures. To explore the possibility of simultaneous confinement of charge carriers (and excitons) and photons within the same individual 1D nanostructure, conceptually the ideal structure would exhibit a Qwof configuration, i.e., a coaxial core-sheath nanostructure with properly engineered size, band-gap alignment, and refractive-index arrangement.

In our attempt to prepare homogeneously alloyed AlGaN nanowires, we accidentally discovered a novel spontaneous phase separation within the $\mathrm{Ga}-\mathrm{Al}-\mathrm{N}$ alloy nanowire system. The GaN/AlGaN core-sheath heterostructured nanowires were prepared using a chemical vapor transport process, ${ }^{11}$ i.e., by transporting gallium and/or aluminum chloride $\left(\mathrm{AlCl}_{3}\right)$ onto the nickel-coated sapphire substrate under a flow of ammonia $\left(\mathrm{NH}_{3}\right)$. It was also found that simultaneous doping could be readily achieved, along with the spontaneous phase separation of GaN/ $\mathrm{Al}_{x} \mathrm{Ga}_{1-x} \mathrm{~N}$ core - sheath heterostructures. For example, manganese-doped $\mathrm{GaN} / \mathrm{Al}_{x} \mathrm{Ga}_{1-x} \mathrm{~N}$ heterostructured nanowires were prepared by transporting manganese chloride $\left(\mathrm{MnCl}_{2}\right)$ and gallium onto nickel-coated sapphire substrates under a flow of $\mathrm{NH}_{3}$. One important advantage of this manganese doping is that the resultant core-sheath nanostructures seem to be much smoother than those without manganese addition (see Supporting Information) for an unknown reason. Scanning electron microscopy (SEM) observation reveals a large quantity of nanowires with diameters up to hundreds of nanometers and lengths up to several micrometers (Figure 1A). The nanowires exhibit flat, quasi-faceted end surfaces. Bright-field transmission electron microscopy (TEM) images of the nanowire show a "dark" core and a "bright" sheath along the axis of the nanowires (Figure 1B, C) with smooth surfaces and sharp interfaces. The cores have diameters in the range of $5-40 \mathrm{~nm}$, whereas the sheath thickness is in the range of 50-200 nm. The selected 

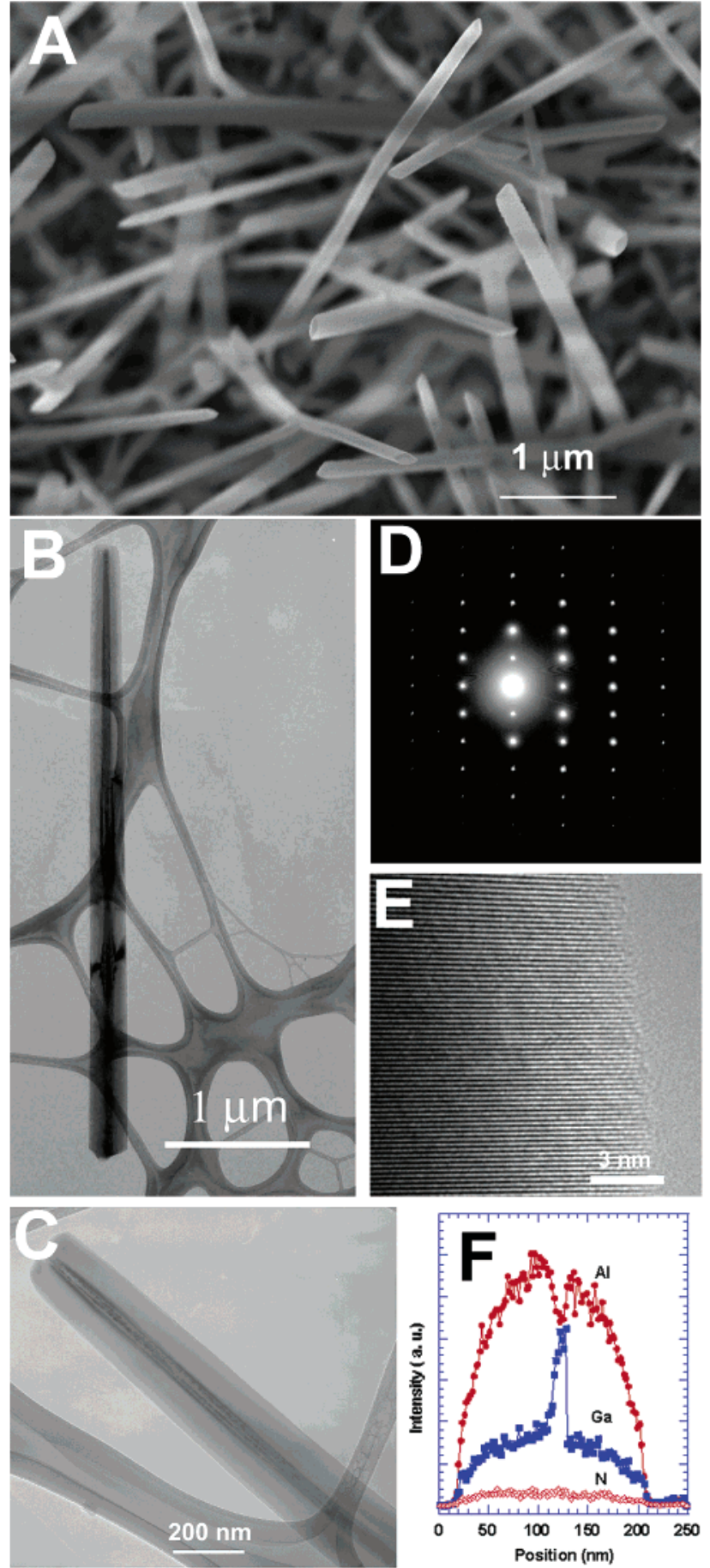

Figure 1. (A) SEM image of manganese-doped $\mathrm{Al}_{x} \mathrm{Ga}_{1-x} \mathrm{~N} / \mathrm{Ga}$ nanowires. (B, C) TEM images of nanowires. (D) Electron diffraction pattern taken along the [100] zone axis. (E) HRTEM image of the $\mathrm{Al}_{x} \mathrm{Ga}_{1-x} \mathrm{~N}$ sheath. (F) Compositional line profile across a nanowire (red open circles denote data for nitrogen, blue solid boxes denote data for gallium, and red solid circles denote data for aluminum). The nanowires were synthesized using a nickel catalyst that was deposited onto a sapphire substrate through direct reaction of the gallium metal and $\mathrm{NH}_{3}$ at $900{ }^{\circ} \mathrm{C}$. Sapphire substrates were cleaned and coated with a layer $(20 \AA)$ of nickel, using a thermal evaporator. The substrates, gallium and $\mathrm{AlCl}_{3}$, were transferred into a small quartz tube. The substrates were typically placed $3 \mathrm{~cm}$ away from the gallium metal, which is also placed $3 \mathrm{~cm}$ away from the $\mathrm{AlCl}_{3}$. The quartz tube was then placed inside a furnace quartz tube, with the substrates placed downstream of the $\mathrm{NH}_{3}$ flow. The temperature of the furnace was ramped to $900{ }^{\circ} \mathrm{C}$ at a rate of $100{ }^{\circ} \mathrm{C} / \mathrm{min}$ and maintained for $10 \mathrm{~min}$ under a constant flow of $\mathrm{NH}_{3}(20 \mathrm{sccm})$. In case of manganese doping, the same conditions were used, except that $\mathrm{MnCl}_{2}$ was transferred into the small quartz tube.
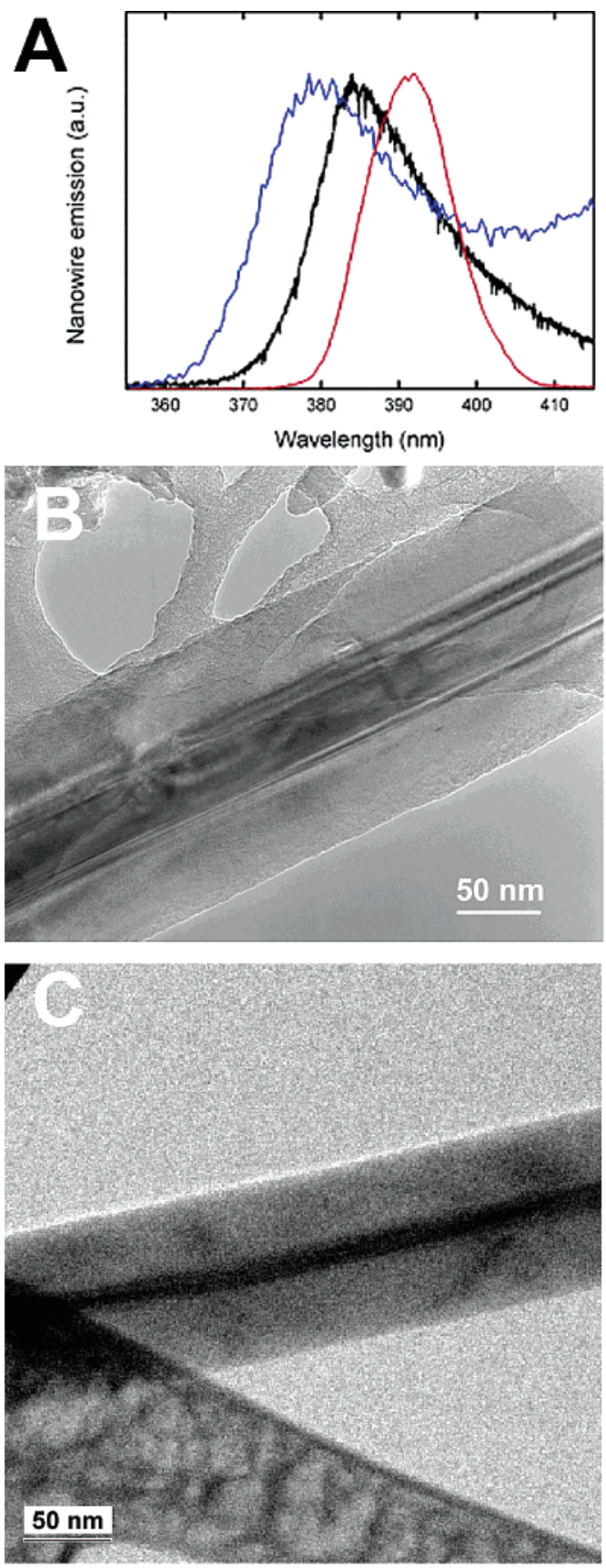

Figure 2. (A) Photoluminescence (PL) spectra of the heterostructure nanowires with core sizes of $20 \mathrm{~nm}$ (blue line), $52 \mathrm{~nm}$ (black line), and the GaN nanowires (red line). (B) TEM image of the heterostructure nanowire with a core size of $52 \mathrm{~nm}$. (C) TEM image of the heterostructure nanowire with a core size of $20 \mathrm{~nm}$. PL measurements were made with an unpolarized $\mathrm{He}-\mathrm{Cd}$ laser, operating at $325 \mathrm{~nm}$.

area electron diffraction that was recorded for the entire coresheath nanowire displays a single pattern (Figure 1D), indicating that the heterostructured nanowires are monolithically single crystalline. High-resolution TEM images of the nanowires (Figure 1E) further confirm the single-crystalline nature of these nanowires. More importantly, energy-dispersive X-ray spectroscopy (EDX) line profiles of the elemental composition across the nanowires (Figure 1F) unambiguously show that these heterostructured nanowires are comprised of thin GaN cores and $\mathrm{Al}_{0.73} \mathrm{Ga}_{0.27} \mathrm{~N}$ sheaths. For those samples that were prepared with the addition of $\mathrm{MnCl}_{2}, \mathrm{EDX}$ analysis shows that $\sim 2 \%$ of the manganese has been uniformly doped into the nanowire lattices. 

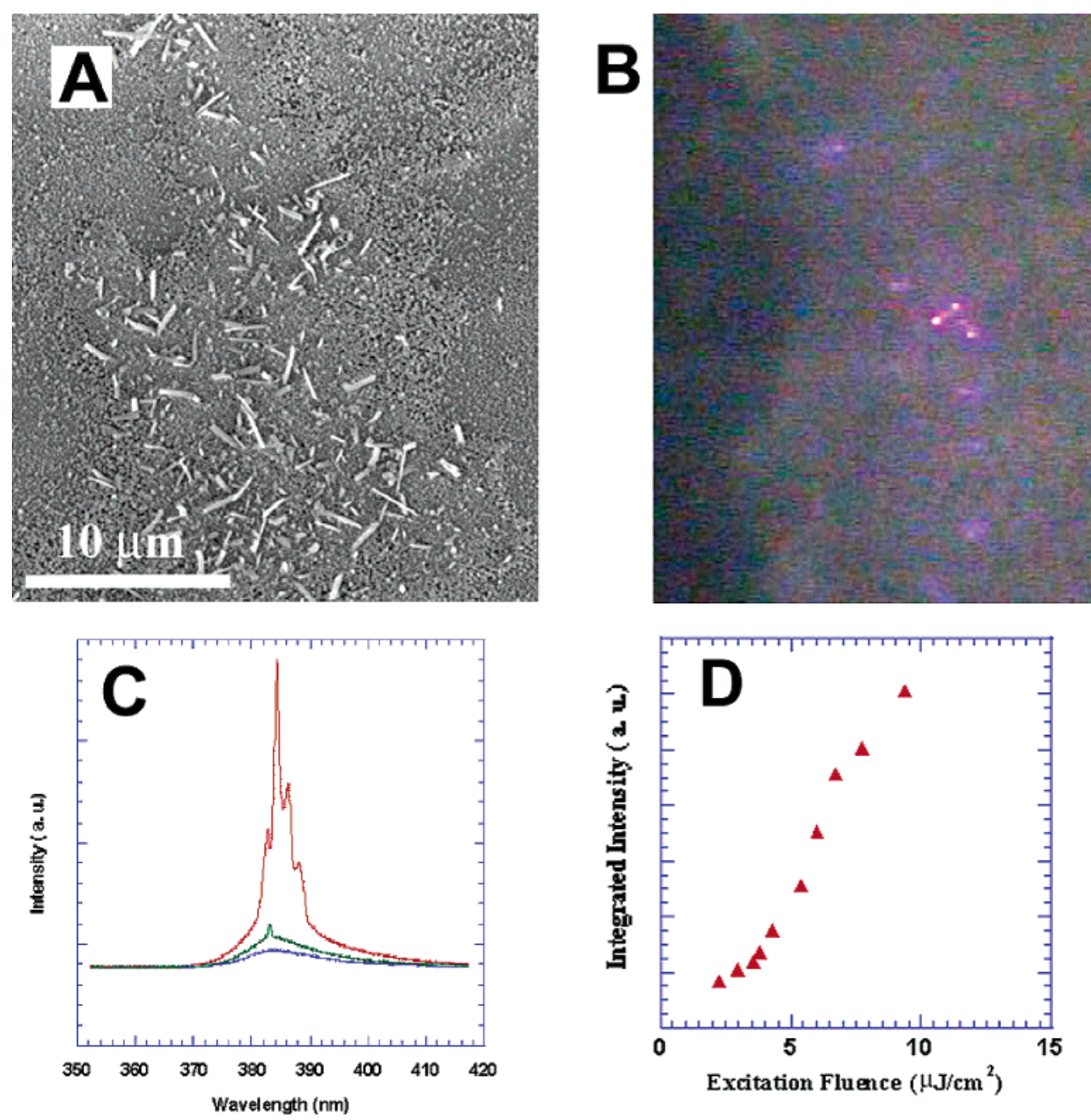

Figure 3. (A) SEM image of as-grown nanowires on a substrate and (B) a corresponding far-field optical image of the lasing emission. (C) Light-emission spectra below (blue), near (green), and above (red) the threshold $\left(\sim 2-3 \mu \mathrm{J} / \mathrm{cm}^{2}\right)$. (D) Power dependence of the output integrated emission intensity. The pulsed UV radiation $(310 \mathrm{~nm}, 4.0 \mathrm{eV})$ is derived from the frequency-quadrupled output of an optical parametric amplifier (OPA). The OPA is seeded with regeneratively amplified light at $800 \mathrm{~nm}$, from a home-built Ti:sapphire oscillator (88 MHz) and a commercial regen/bowtie amplifier $(1 \mathrm{kHz}, 2.5 \mathrm{~mJ})$. The UV pulses are $\sim 100-200 \mathrm{fs}$ in duration, with pulse energies of $2-3 \mu \mathrm{J}$. The pulses are attenuated with a variable neutral density filter, and the UV is spectrally isolated using a black glass band-pass filter (Edmund, model UG11). The beam is directed to the far-field microscope setup. The beam is focused obliquely onto the sample with a $\mathrm{CaF}_{2}$ lens. The spot size is kept relatively large (typically $200 \mu \mathrm{m} \times 100 \mu \mathrm{m})$, to aid in uniform illumination of the nanowires. The light emitted from the sample is collected by a microscope objective (Olympus, NA 0.7) and is imaged by a video camera. The spatial resolution of the microscope is $\sim 0.5-1 \mu \mathrm{m}$. A removable mirror redirects the emission to an optical fiber, which is routed to a spectrograph, and a charge-coupled device (CCD, Roper Scientific) is used for spectra collection.

The single-crystalline nature, structurally perfect conjunction of the interface, and the one-step fabrication process for these nanowires imply that the heterostructure is formed by a selforganization process through a spontaneous phase separation in the $\mathrm{Al}-\mathrm{Ga}-\mathrm{N}$ ternary alloy system. Strain-induced selforganization of alloyed material systems has proved useful for the preparation of superlattice or heterostructures at a nanometer scale and has been already extensively utilized in zero- and twodimensional systems. ${ }^{12-15}$ For example, strained GaN quantum dot arrays have been prepared within an AlN matrix. ${ }^{13}$ Straininduced self-ordering has also been used to prepare arrays of quantum wire lasers in the AlGaInP system on a planar substrate. ${ }^{12}$ Self-organization of these quantum dot/wire/well systems is driven principally by the stresses that are generated from the lattice mismatch between the substrate and the materials, and/or the end member of the alloy system. ${ }^{16-18}$ The free-standing nature of the nanowires should allow us to safely disregard the effect from the substrate. We note, however, that the lattice mismatch between the end-member components of the $\mathrm{Ga}-\mathrm{Al}-\mathrm{N}$ alloy system (i.e., $\mathrm{GaN}$ and $\mathrm{AlN}$ ) is quite small $(2.5 \%)$; hence, thermodynamically, the spontaneous phase separation will not be favored at room temperature. ${ }^{19}$ Although the GaN nanowire growth is driven by the well-defined vaporliquid-solid (VLS) mechanism, as revealed by the necessity for nickel catalysts during the growth, ${ }^{20}$ the stresses that are generated at the curved surfaces of the nanowires, which are expected to be significant as the size approaches the nanometer scale, might be the principal driving force of the presently observed self-ordering.

The curved surfaces of materials are subjected to a local pressure $\Delta P$ (and, in turn, stresses), according to the Laplace equation: $\Delta P=\gamma\left[\left(1 / r_{1}\right)+\left(1 / r_{2}\right)\right]$, where $r_{1}$ and $r_{2}$ are two principal radii of curvature in a given point of a surface and $\gamma$ is the surface tension. For nanowires, $r_{1}$ and $r_{2}$ correspond to the curvature in the radial and longitudinal directions, respectively; the former equals the radius of the nanowires, whereas the latter can be assumed to be infinite. Therefore, $\Delta P$ generated on the curved surface of the nanowires can be estimated as $\Delta P$ $=\gamma / r_{\text {nanowire }}$, where $r_{\text {nanowire }}$ is the nanowire radius. Because of the positive curvature of the surfaces, compressive stresses in the inward radial direction arise and become significant as the size decreases. ${ }^{21}$ Similar phenomena can be found in the 

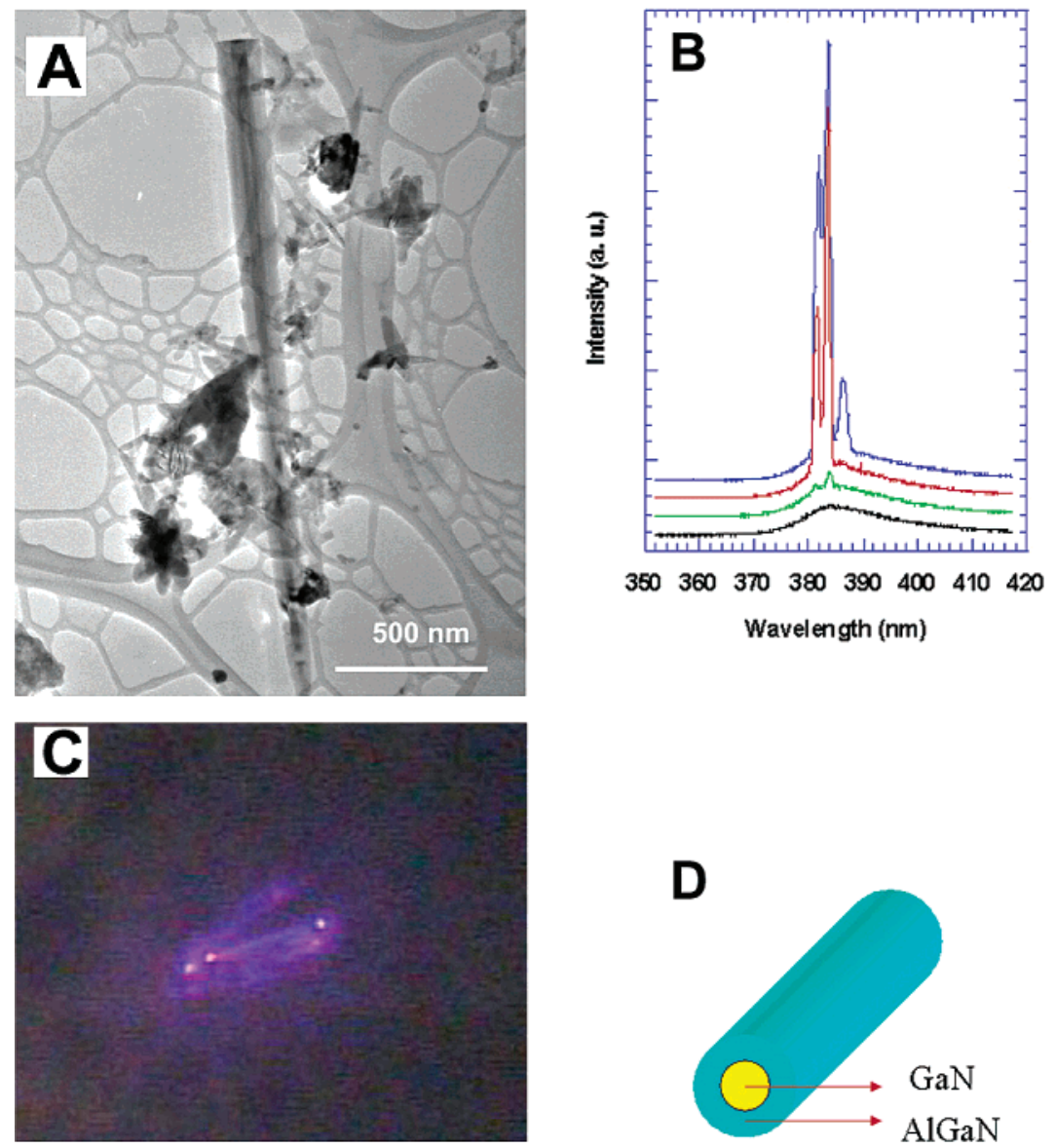

Figure 4. (A) TEM image of as-grown nanowires on a TEM grid and (B) PL and lasing spectra of individual nanowires with a core size of $52 \mathrm{~nm}$, excited by a pulse of 26 (black), 28 (green), 33 (red), and $40 \mu \mathrm{J} / \mathrm{cm}^{2}$ (blue). (C) Typical far-field optical image of the individual core-sheath nanostructure on the TEM grid, showing the waveguided emission. (D) Schematic of the GaN/AlGaN core-sheath nanostructures. For measurement of individual nanowire PL emission, nanowires were dispersed on carbon-coated TEM grids. Far-field images and spectra were collected for the area showing PL in the grids; the same area was subsequently analyzed via TEM.

overgrowth of semiconductor alloy thin films, where the spontaneous phase separation is accompanied by surface roughening (i.e., the formation of curved surfaces). ${ }^{14-18}$ Such a strain-induced self-ordering process might have also led to the irregular spatial compositional modulation in the GaInAs nanowire system that was reported previously by the Buhro group. ${ }^{22}$ Recently, it has also been proposed that even the pure GaN nanowire can experience significant biaxial compressive stress. $^{23}$

Importantly, these core-sheath GaN/AlGaN nanowires provide unique model systems for the study of simultaneous photon and carrier confinement. The GaN core size is comparable to the Bohr radius of the exciton for $\mathrm{GaN}(11 \mathrm{~nm}) .^{24}$ The band gap of the core $(\mathrm{GaN}, 3.42 \mathrm{eV})$ is smaller than that of the sheath $\left(\mathrm{Al}_{0.75} \mathrm{Ga}_{0.25} \mathrm{~N}, 5.25 \mathrm{eV}\right)$, i.e., a type-I band-offset alignment. Both factors would lead to effective carrier/exciton confinement within such unique core-sheath nanostructures. To test this hypothesis, the photoluminescence (PL) of single heterostructured nanowires was characterized. Significantly, blue-shifted PL, which is a signature of the quantum confinement effect, was observed. Figure 2A shows the PL spectra taken from individual core-sheath nanowires with core sizes of $52 \mathrm{~nm}$ (Figure 2B) and $20 \mathrm{~nm}$ (Figure 2C), and GaN nanowires with a diameter of $200 \mathrm{~nm}$. The PL emission from the 200-nm nanowires can be considered to be equivalent to the bulk bandedge emission. The PL of the heterostructure nanowires with core sizes of 52 and $20 \mathrm{~nm}$ is blue-shifted to 385 and $379 \mathrm{~nm}$, respectively. Moreover, in coaxial heterostructures nanowires, modulation doping of larger band-gap material will allow spatial separation of ionized dopants and free carriers, and, thus, higher mobilities could be achieved. Indeed, measurement of the electrical conductance of these manganese-doped nanowires showed that both the sheath and core have $p$-type electrical characteristics. Furthermore, the GaN core typically exhibits a very low resistivity $(\sim 0.2 \Omega \cdot \mathrm{cm}$; see Supporting Information) that is comparable to that of heavily doped silicon and GaN. These results suggest that carriers (i.e., holes) are confined in the $\mathrm{GaN}$ core or at the interfaces in these core-sheath nanowires. No experimental optimization, however, has been made on the doping of such core-sheath structures.

In addition to the carrier/excitonic confinement within the GaN core region, there are two additional characteristics of the nanostructures that would allow us to examine the nature of photonic confinement (waveguiding and amplification) within such structures. First, the refractive index of the sheath $(2.25$ for $\left.\mathrm{Al}_{0.75} \mathrm{Ga}_{0.25} \mathrm{~N}\right)$ is smaller than that of the core region $(2.54$ for $\mathrm{GaN}) .{ }^{25}$ The core-sheath structure essentially resembles a properly index-engineered optical fiber. Indeed, with this arrangement of refractive index and size, the nanostructure would behave as a single-mode optical fiber for UV light. Second, the entire core-sheath structure has well-defined end surfaces (which become natural mirrors for the optical fiber and form an optical cavity). Hence, this structure is an ideal Qwof nanostructure, which would, for the first time, allow us to examine the possibility of simultaneous confinement of the exciton and the photon (followed by their possible amplification) 
within the same chemically synthesized nanostructure. To examine this possibility, individual core-sheath nanostructures were optically pumped and their power-dependent PL was collected. Figure 3A shows an SEM image of the as-grown nanowires on the substrate, and the corresponding far-field optical image of nanowire emission is shown in Figure 3B. The localization of bright emission near the nanowire ends indicates stimulated emission from the nanowires. Figure 3C shows a series of far-field spectra taken as a function of the pump fluence on these nanowires. Below the lasing threshold, the PL spectrum is broad and featureless. Near and above the threshold (at $\sim 2-3$ $\left.\mu \mathrm{J} / \mathrm{cm}^{2}\right)$, sharp features that are centered at $\sim 384 \mathrm{~nm}(\sim 3.2 \mathrm{eV})$ appear in the spectrum, indicating the onset of lasing emission from the $\mathrm{GaN}$ core of nanowires. Power-dependent lasing spectra near the threshold reflects the typical lasing action of a wide band-gap semiconductor (Figure 3D).

Individual nanowire exhibits similar lasing emission behavior. Figure 4A shows a TEM image of such a core-sheath structure supported on a TEM grid for far-field emission study. The core size of this nanowire is $52 \mathrm{~nm}$. Figure 4B shows a series of spectra taken as a function of pump fluence, whereas Figure 4C shows a typical far-field emission image from the coresheath nanowires. Sharp features centered at $\sim 384 \mathrm{~nm}$ similarly appear above the threshold (in this case, $\sim 28 \mu \mathrm{J} / \mathrm{cm}^{2}$ ), indicating the onset of lasing emission. The measured thresholds $P_{\mathrm{t}}$ for the heterostructure nanowires are higher than that observed in GaN nanowires by almost an order of magnitude, although they are still lower than those reported for many other types of GaN microstructures, ${ }^{26-28}$ which may be due to the reduction in gain volume. Indeed, the volume of the $\mathrm{GaN}$ core in these heterostructure nanowires is smaller by several orders than that of the other $\mathrm{GaN}$ nanowires or $\mathrm{GaN}$ microstructure lasing cavities. In this regard, the lasing characteristics of the $\mathrm{GaN}$ core indicate that these heterostructure nanowires are very efficient gain media, which can be attributed to the ideal photon confinement structures. In the radial direction, the $\mathrm{Al}_{x} \mathrm{Ga}_{1-x} \mathrm{~N}$ sheath has a lower refractive index and, thus, can act as a cladding layer for the waveguide to confine the photon. In addition, the spontaneously organized interfaces have fewer defects, which can otherwise degrade the lasing emission. As a comparison, it must be emphasized that no waveguiding or lasing has been observed thus far for pure $\mathrm{GaN}$ nanowires with diameters of $<100 \mathrm{~nm}$.

Taken together, the results presented here imply that the spontaneous phase separation in the nanowires could be driven by the intrinsic stresses. Such a self-ordering process might be a rather general phenomenon in the low-dimensional systems at the nanometer scale and, therefore, could be utilized for the preparation of well-defined, complex structures of nanomaterials. Our characterization of the core-sheath $\mathrm{Al}-\mathrm{Ga}-\mathrm{N}$ nanostructures suggests that this approach can produce multifunctional building blocks that possess unique optical and electronic properties. For example, these heterostructure nanowires may be used as building blocks for optoelectronic and electronic devices by utilizing their capability of simultaneous photon, exciton, and carrier confinement, which have led to the first GaN-based quantum wire UV lasers described here.

Acknowledgment. This work was supported by the Camille and Henry Dreyfus Foundation, research Corporation, the
Hellman Family Faculty Foundation, the Beckman Foundation, and the National Science Foundation and Department of Energy. P.Y. is an Alfred P. Sloan Research Fellow. Work at the Lawrence Berkeley National Laboratory was supported by the Office of Science, Basic Energy Sciences, Division of Materials Science, U.S. Department of Energy. We thank the National Center for Electron Microscopy for the use of their facilities.

Supporting Information Available: TEM images and compositional profiles for pure $\mathrm{Al}_{x} \mathrm{Ga}_{1-x} \mathrm{~N} / \mathrm{GaN}$ core-sheath heterostructure nanowires, optical image of the visible and UV emission from the sample shown in Figure 3A, and temperaturedependent $I-V$ electrical transport data on core-sheath nanowire (PDF). This material is available free of charge via the Internet at http://pubs.acs.org.

\section{References and Notes}

(1) Kapon, E., Ed. Semiconductor Lasers I: Fundamentals; Academic Press: San Diego, CA, 1999.

(2) Klimov, V. I.; Mikhailovsky, A. A.; Xu, S.; Malko, A.; Hollingsworth, J. A.; Leatherdale, C. A.; Eisler, H. J.; Bawendi, M. G. Science 2000, 290, 314 .

(3) Kapon, E. Proc. IEEE 1992, 80, 398

(4) Hayamizu, Y.; Yoshita, M.; Watanabe, S.; Akiyama, H.; Pfeiffer, L. N.; West, K. W. Appl. Phys. Lett. 2002, 81, 4937.

(5) Nakamura, S. Mater. Res. Bull. 1997, 22, 29

(6) Monemar, B.; Pozina, G. Prog. Quantum Electron. 2000, 24, 239.

(7) Pearton, S. J.; Ren, F. Adv. Mater. 2000, 12, 1571.

(8) Yu, E. T., Manasreh, M. O., Eds. III-V Nitride Semiconductors. Applications and Devices; Optoelectronic Properties of Semiconductors and Superlattices 16; Taylor and Francis: New York, 2003.

(9) Huang, M.; Mao, S.; Feick, H.; Yan, H.; Wu, Y.; Kind, H.; Weber, E.; Russo, R.; Yang, P. Science 2001, 292, 1897.

(10) Johnson, J.; Yan, H.; Schaller, R.; Haber, L.; Saykally, R.; Yang, P. J. Phys. Chem. B 2001, 105, 11387.

(11) Johnson, J.; Choi, H. J.; Knutsen, K. P.; Schaller, R. D.; Saykally, R. J.; Yang, P. Nat. Mater. 2002, 1, 101.

(12) Pearah, P. J.; Chen, A. C.; Moy, A. M.; Hsieh, K.; Cheng, K. IEEE J. Quantum Electron. 1994, 30, 608.

(13) Widmann, F.; Simon, J.; Daudin, B.; Feuillet, G.; Rouviere, J. L.; Pelekanos, N. T.; Fishman, G. Phys. Rev. B 1998, 58, R15989.

(14) Chou, S. T.; Cheng, K. Y.; Chou, L. J.; Hsieh, K. C. Appl. Phys. Lett. 1995, 66, 2220.

(15) Vennegues, P.; Lahreche, H. Appl. Phys. Lett. 2000, 77, 4310.

(16) Tersoff, J.; Teichert, C. M.; Lagally, G. Phys. Rev. Lett. 1996, 76, 1675 .

(17) Petroff, P. M.; Cho, A. Y.; Reinhart, F. K.; Gossard, A.; Wiegmann, C. W. Phys. Rev. Lett. 1982, 48, 170.

(18) Singh, R.; Dopplapudi, D.; Moustakas, T. D. Appl. Phys. Lett. 1997, $70,1089$.

(19) Takayama, T.; Yuri, M.; Itoh, K.; Baba, T.; Harris, J. S., Jr. Jpn. J. Appl. Phys. 2000, 39, 5075.

(20) Wu, Y.; Yang, P. J. Am. Chem. Soc. 2001, 123, 3165.

(21) Schweigert, I. V.; Lehtinen, K. E. J.; Carrier, M. J.; Zachariah, M R. Phys. Rev. B 2002, 65, 235410.

(22) Markowitz, P. D.; Zach, M. P.; Gibbons, P. C.; Penner, R. M.; Buhro, W. E. J. Am. Chem. Soc. 2001, 123, 4502.

(23) Seo, H. W.; Bae, S. Y.; Park, J.; Yang, H. N.; Park, K. S.; Kim, S. J. Chem. Phys. 2002, 116, 9492.

(24) Preschilla, N.; Major, S.; Kumar, N.; Samajdar, I.; Srinivasa, R. S. Appl. Phys. Lett. 2000, 77, 1861.

(25) Webb-Wood, G.; Özgür, Ü.; Everitt, H. O.; Yun, F.; Morkoç, H. Phys. Status Solidi A 2001, 188, 793.

(26) Tanaka, S.; Hirayama, H.; Aoyagi, Y.; Narukawa, Y.; Kawakami, Y.; Fujita, S.; Fujita, S. Appl. Phys. Lett. 1997, 71, 1299.

(27) Schmidt, T. J.; Cho, Y.; Song, J. J.; Yang, W. Appl. Phys. Lett. 1999, 74, 245

(28) Yang, X. H.; Schmidt, T. J.; Shan, W.; Song, J. J.; Godenberg, B. Appl. Phys. Lett. 1995, 66, 1. 